This item was submitted to Loughborough's Research Repository by the author.

Items in Figshare are protected by copyright, with all rights reserved, unless otherwise indicated.

\title{
Flight testing Boustrophedon coverage path planning for fixed wing UAVs in
} wind

PLEASE CITE THE PUBLISHED VERSION

PUBLISHER

IEEE

VERSION

AM (Accepted Manuscript)

\section{PUBLISHER STATEMENT}

(C) 2019 IEEE. Personal use of this material is permitted. Permission from IEEE must be obtained for all other uses, in any current or future media, including reprinting/republishing this material for advertising or promotional purposes, creating new collective works, for resale or redistribution to servers or lists, or reuse of any copyrighted component of this work in other works.

\section{LICENCE}

All Rights Reserved

\section{REPOSITORY RECORD}

Coombes, Matthew, Wen-Hua Chen, and Cunjia Liu. 2019. "Flight Testing Boustrophedon Coverage Path Planning for Fixed Wing Uavs in Wind". figshare. https://hdl.handle.net/2134/37149. 


\title{
Flight Testing Boustrophedon Coverage Path Planning for Fixed Wing UAVs in Wind
}

\author{
Matthew Coombes, Wen-Hua Chen, Cunjia Liu \\ Department of Automotive and Aeronautical Engineering \\ Loughborough University, \\ Loughborough, LE11 3TU UK
}

\begin{abstract}
A method was previously developed by this author to optimise the flight path of a fixed wing UAV performing aerial surveys of complex concave agricultural fields. This relies heavily on a flight time in wind prediction model as its cost function. This paper aims to validate this model by comparing flight test results with the model prediction. There are a number of assumptions that this model relies on. The major assumption is that wind is steady and uniform over the small area and time scales involved in a survey. To show that this is reasonable, wind fields measurements will be taken from a multi rotor UAV with an ultrasonic windspeed sensor.
\end{abstract}

Index Terms-Aerial Surveying, Coverage Path Planning, Remote Sensing, Boustrophedon paths, Wind, Trochoids.

\section{INTRODUCTION}

Currently the world population is booming, the world bank states that at the current rate of growth we will need to produce $50 \%$ more food by 2050 [1]. To achieve this, farming needs to become more efficient with the use of precision agriculture. The collection of agricultural field data is a major part of this, e.g. Soil water saturation, crop yield, crop health. This data can then in turn be turned into actionable information, e.g. variable rate fertilizer of pesticide application.

This data is normally painstakingly collected by hand, however the use of remote sensing is changing all that. By analysing multi-spectral imagery from satellites, some of these measurements can be performed much faster and cheaper [2]. However satellites have low resolution, low persistence, due to their fixed orbit, images can also be blocked by cloud, or haze. The alternative is to use a UAV (Unmanned Aerial Vehicle) to traverse the ROI (Region of Interest) and take multiple aerial images that cover the whole ROI. These images are stitched together using structure from motion photogrammetry software into a geo-referenced high resolution orthomosaics or Digital Elevation Model (DEM) [3]. UAV's are shown to be a very successful tool for precision agriculture, for example; disease mapping [4], or yield prediction in barley [5].

These UAV's need to be flown in such a way that will ensure total coverage of the field. This is called Coverage Path Planning (CPP). The most efficient method to ensure coverage of a convex polygon is a simple lawn mower pattern path aka a Boustrophedon path [6], [7]. This CPP method is illustrated in Fig. 1, where the back and forth paths and the total image coverage is shown. However in a study of Finnish agricultural fields only $13 \%$ where a simple convex

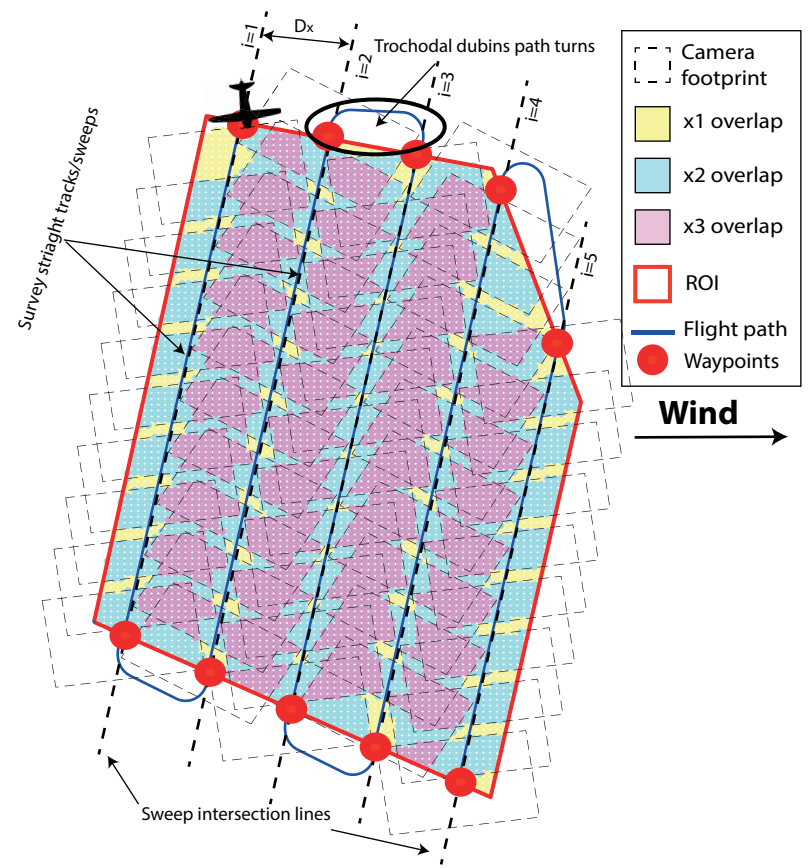

Fig. 1: Example Boustrophedon path of a convex polygon, showing the camera image footprints giving complete coverage. Sidelap $40 \%$, overlap $40 \%$

shape [8]. This means that a more complex mission planner is required to handle the more common complex concave field shapes. There exists a number of commercial mission planning tools that let the user select a ROI to survey and auto generate a survey path based on the vehicle and survey requirements (Camera used, required image overlap) [9][11]. However as explained in [12] these are inadequate as they simply plot a Boustrophedon path around a concave field's convex hull, which makes for a wastefully long path. This is why many CPP methods in literature decompose the concave polygon into a number of smaller convex ones and plot a Boustrophedon path around each polygon [8], [13][15].

Small Fixed wing survey UAVs are nonholonomic vehicles, and due to their size and speed are heavily effected by the wind. This requires a more bespoke method for efficiently planning their surveys, as factors previous ignored are much more critical. [7] shows how sensitive to wind small UAVs are, where over a $22 \mathrm{~min}$ real UAV aerial survey a $7 \mathrm{~m} / \mathrm{s}$ wind increased the survey time by around $20 \%$. This time 
increase is dependent on the relative wind direction to the sweeps, where flying perpendicular to the wind minimises flight time, as proven in [16]. Being able to account for the wind is needed to properly minimise flight time. Two promising polygon decomposition based mission planning techniques use simplified cost functions. One uses number of turns [14], and the other tries to minimise the sum of long axis length from each decomposed convex polygon [15]. These heuristics do a reasonable job of approximating the survey time, however as they don't account for wind these method lose their optimality in real life.

There has not been much work looking at optimising aerial survey mission planning in wind. [17] provides a mission planning method for minimising fixed wing flight time UAV in a uniform wind field for aerial surveys. It plots a full Boustrophedon path in the same orientation across the whole concave field polygon, then calculates a time cost in wind for each sweep and uses a modified travelling salesman solver to find the fastest order and direction to traverse all the sweeps without forcing the aircraft to perform manoeuvres that it is incapable of. The previous work by this author presents a similar flight time model for calculating flight time for each portion of the flight in wind [18]. This is then extended too more complex concave fields by optimally decomposing the concave polygon into multiple simple convex polygons, which can be fully imaged using a Boustrophedon path [16]. These, as well as many other papers on other mission and path planning applications, make the assumption of a steady uniform wind [19]-[23].

However, as in a real survey, we can not assume perfect path following or steady wind. No other work has attempted to validate their wind model, or their steady uniform wind assumption in real world tests. This paper aims to address this, not only to validate this authors proposed model but also to be used to show that over a small area and over a short time the wind can be assumed to be steady and uniform. This is an important untested assumption that many similar works relies on. Two sets of flight tests are performed: multiple flights with a real survey UAV flying a Boustrophedon coverage path to compare the Flight Time in Wind (FTIW) model to real survey flights. The second uses rotary wing platform with an ultrasonic wind speed sensors to map the wind field around the survey area taking spot wind measurements to show that the wind is approximately steady and uniform. The second are multiple flights with a real survey UAV flying a Boustrophedon coverage path to compare the FTIW model to real survey flights.

The remainder of this paper is organised as follows; Section II gives an overview of the FTIW model. In Section III discusses the experimental setup, the airframes and systems to fly the survey and to measure the wind field. In the penultimate Section IV, the results of the surveys and wind field measurements are presented and discussed. Finally in Section $\mathrm{V}$ concluding remarks are made.

\section{Coverage Path Planning}

A Boustrophedon path consists of back and forth motions at a particular survey angle $\left(\psi_{s}\right)$ across a ROI. The path consists of two different states of flight; the straight sweep paths where the images are captured, and the turn manoeuvre used to transition between sweeps. This is laid out in detail in [18], and as such, will only have a brief overview here.

The distance between sweeps $\left(D_{x}\right)$ is set based on camera parameters, required Ground Sample Distance (GSD) and \% image sidelap $\left(w_{s}\right)$ shown in Eq (1). Perfect path following is assumed, for both flight time calculation, and image coverage. We assume a user selected constant airspeed is maintained by the aircraft's autopilot, as well as the windspeed and direction being known before the flight when the mission is generated. This can be taken from local weather stations or ground measurements.

$$
D_{x}=2 \tan \left(\frac{\text { fov }_{x}}{2}\right) \frac{\left(1-w_{s}\right) N_{x} G S D}{\text { fov }_{x}}
$$

where $G S D$ is in $\frac{m}{p i x}$, fov $_{x}$ is the horizontal angular Field Of View (FOV) of the sensor, $N_{x}$ is the number of pixels the sensor has in the horizontal direction. The height of the vehicle is not included in this equation as it is encapsulated in the $G S D$.

The $\psi_{s}$ is fixed based on the angle of the long axis of the convex polygon. This is a standard technique to minimise flight time by minimising turns [14].

\section{A. Flight Time Model in Wind}

Wind can have a significant effect on small aircraft; the windspeed experienced by a small UAV can easily be $50 \%$ of the airspeed. As a result, it is vital to account for wind for small survey UAVs. This section details how the flight time is calculated for each part of the flight.

1) Sweep Paths: To find the time to fly along a sweep line $\left(t_{s}\right)$ is a case of the length of the sweep $L_{s}^{i}$ divided by the aircraft's ground speed along it $L_{s} / V_{g}$. The aircraft's ground speed can be calculated by wind triangle vector subtraction. To get the total flight time for all sweeps, the time for each sweep with index $i$ (sweep example $i$ indexing shown in Fig. 1) needs to be summed as shown in Eq (2). In this Equation $n_{s}$ is the number of sweeps. As every other sweep is performed in the opposite direction when $i$ is even the heading rotated by $180^{\circ}$.

2) Turns: The turns between sweeps are Dubins Paths extended to account for the wind. Dubins Paths form a continuous paths between a start and end point from two turn circles and a tangent between them. In order to account for wind these circles are now trochoids. This represents a turn circle in a moving frame of reference i.e. the wind. The time taken for this full turn manoeuvre $\left(t_{t}\right)$ consists of the time taken to fly each trochoidal turn $\left(t_{1}, t_{2}\right)$, and the straight tangent between them, shown in Fig. 2 and the equation is presented below in Eq (3). In this Equation $L_{x t}$ is the length of the tangent in $\mathrm{x}$ and $L_{y t}$ is the length in the $\mathrm{y}$ direction. $t_{1}$ and $t_{2}$ are generated by finding a tangent between them 


$$
t_{s}=\sum_{i=1}^{n_{s}}\left\{\begin{aligned}
\text { if } i \text { odd } & \left(\frac{L_{s}^{i}}{\left.V \cos \left(\operatorname{asin}\left(\frac{V_{w}}{V} \sin \left(\psi_{w t a}\right)\right)\right)\right)+\left(V_{w} \cos \left(\psi_{w t a}\right)\right.}\right) \\
\text { if } i \text { even } & \left(\frac{L_{s}^{i}}{\left.V \cos \left(\operatorname{asin}\left(\frac{V_{w}}{V} \sin \left(\psi_{w t a}+\pi\right)\right)\right)\right)+\left(V_{w} \cos \left(\psi_{s}+\pi\right)\right.}\right)
\end{aligned}\right.
$$

that creates a feasible flight path. The equations to calculate these are laid out in [19] and [18].

\section{SURVEY FLIGHT TEST METHOdOLOGY}

In order to assess the flight time prediction from the proposed model, a number of simple survey ROI's and their corresponding CPP paths are defined then flown by an actual survey UAV. Using a known windspeed and direction, the flight time is predicted from the FTIW model then can be compared to the actual flight time. The wind speed and direction used for calculations are obtained from ground measurements immediately before the flights. These surveys were repeated a number of times in different wind conditions to get a range of results.

A rectangular polygon of $450 \mathrm{X} 250 \mathrm{~m}$ is chosen for the ROI. This is in order to to repeat the same ROI for a different relative wind angles to the sweep direction, there will be three ROIs to fly per mission, rotated about their centre to $330^{\circ}, 285^{\circ}$ and $245^{\circ}$. This will ensure a good range of angles are covered.

For each flight, the aircraft autonomously flies the CPP waypoints shown in Fig. 4 using the L1 adaptive navigation controller available on the autopilot [24]. The desire was to test the survey model in a highly realistic scenario using the exact sort of autopilots, sensors and navigation that will actually be used for surveys by farmers and operators. All three survey ROI's are shown in Fig. 3. Below are the main aircraft and survey parameters required by the model.

$$
\begin{array}{lll}
V=16 \mathrm{~m} / \mathrm{s} & \dot{\psi}=0.7 \mathrm{rad} / \mathrm{s} & D_{x}=77.3 / 49.75 \mathrm{~m} \\
w_{s}=40 \% & h=100 \mathrm{~m} & G S D=0.0234 \frac{\mathrm{m}}{\mathrm{pix}}
\end{array}
$$

While the wind speed and direction can be obtained from weather reports for purposes of pre-planning, the assumption is made that we know accurate wind information beforehand. To show that the assumption of a steady uniform wind holds, the wind field (wind at multiple locations) will be measured at survey altitude by a separate multirotor UAV. The measures wind field will be assessed for spacial and temporal variations. Multiple wind field measurement flight will be flown in different wind conditions. A sweep pattern is flown over the survey area, stopping and hovering at 16 waypoints sampling wind for $15 \mathrm{~s}$.

\section{A. Flight Platforms}

The Skywalker X8 is the fixed wing aircraft used in the survey. Fitted with an autopilot to control all aspects of the aircraft during the survey. The aircraft and its systems can be seen in Fig. 5 and whos major flight parameters are listed below:
The setup consists of an Pixhawk autopilot with PX4 firmware, Pixhawk airspeed sensor, GPS, Raspberry Pi2, Arduino Nano, AeroProbe-Micro-ADC.

Pixhawk is a high-performance autopilot-on-module suitable for fixed wing, multi rotors, helicopters, cars, boats. By intercepting the autopilot telemetry on a Raspberty Pi 2, running Robotic Operating System (ROS) and a ROS package called MAVROS, all the autopilot telemetry data is added to the ROS network as topics. This setup is laid out in greater detail in [25].

In order to perform accurate measurements of the wind, a more accurate airspeed sensor than the low cost sensor that is used by Pixhawk is needed. The sensor chosen was the AeroProbe-Micro-ADC, which is a high accuracy multi hole pitot static probe and data logger.

Using another ROS package called ROSSerial enables the addition of other sensors by interfacing with a Arduino Nano which has a huge range of I/O available that the Raspberry Pi does not. This enables the parsing of the other serial link from AeroProbe which contains the airspeed, Angle of attack, and angle of sideslip data. This data will be published to the ROS network as user defined topics.

All the relevant topics related to airdata, IMU, GNSS, and the AeroProbe are recorded using ROSBags. These can then easily be parsed post process by MatLab's Robotics System Toolbox (RST), and then analysed.

The aircraft that performs the wind field measurements is a DJI S1000 octorotor, with an FT742 ultrasonic wind sensor mounted above the vehicle out of the rotor wash shown in Fig. 6. This allows direct measurement of the wind at any altitude. It has a $15 \mathrm{~min}$ flight time with $520 \mathrm{Wh}$ of batteries, at an all up weight of $5 \mathrm{Kg}$. It has the same ROS based data logging solution for logging location, heading and wind.

\section{SURVEY FLIGHT TEST RESUlTS}

Three separate missions were flown on different days with different wind speeds $\left(7 \mathrm{~m} / \mathrm{s} 225^{\circ}, 7.4 \mathrm{~m} / \mathrm{s} 335^{\circ}, 11 \mathrm{~m} / \mathrm{s} 230^{\circ}\right)$. On each mission there are three rotated ROI to survey. This is to get a range of relative wind directions. One of the surveys will be used as an example to be discussed in detail. All other survey flights results are complied into Table. 2. On the first mission, the S1000 was flown immediately after to access the uniformity of the wind field.

TABLE 1: Parameters for the X8 flying wing

\begin{tabular}{ll}
\hline Parameter & Value \\
\hline Wing Area & $0.734 \mathrm{~m}^{2}$ \\
Aspect Ratio & 7.48 \\
Mass & $2.75 \mathrm{~kg}$ \\
$V_{\text {stall }}$ & $10 \mathrm{~m} / \mathrm{s}$ \\
$V_{\text {cruise }}$ & $16 \mathrm{~m} / \mathrm{s}$ \\
\hline
\end{tabular}




$$
t_{t}=\sum_{i=1}^{n_{s}-1} t_{1}+t_{2}+\left(\frac{\sqrt{L_{x t}^{2}+L_{y t}^{2}}}{V \cos \left(\operatorname{asin}\left(\frac{V_{w}}{V} \sin \left(\operatorname{atan} \frac{L_{x t}}{L_{y t}}\right)\right)\right)+V_{w} \cos \left(\operatorname{atan} \frac{L_{x t}}{L_{y t}}\right)}\right)
$$

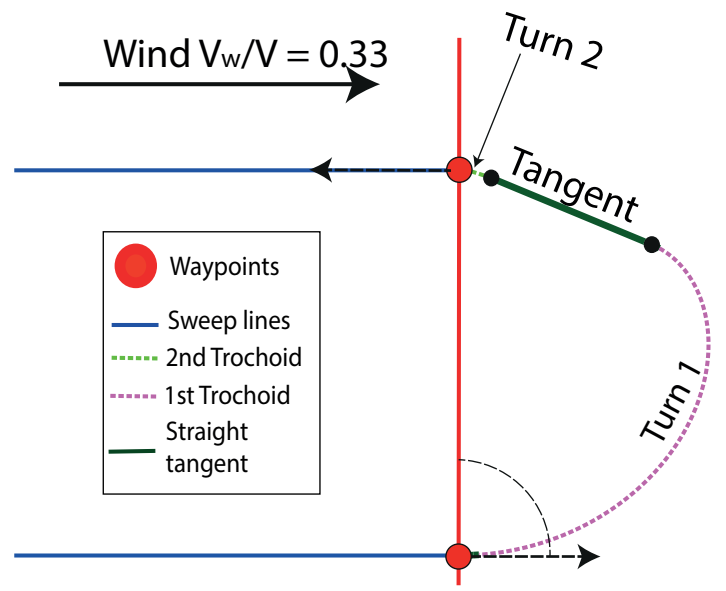

Fig. 2: Trochoidal turn manoeuvre to transit between one straight line survey sweep to the next.

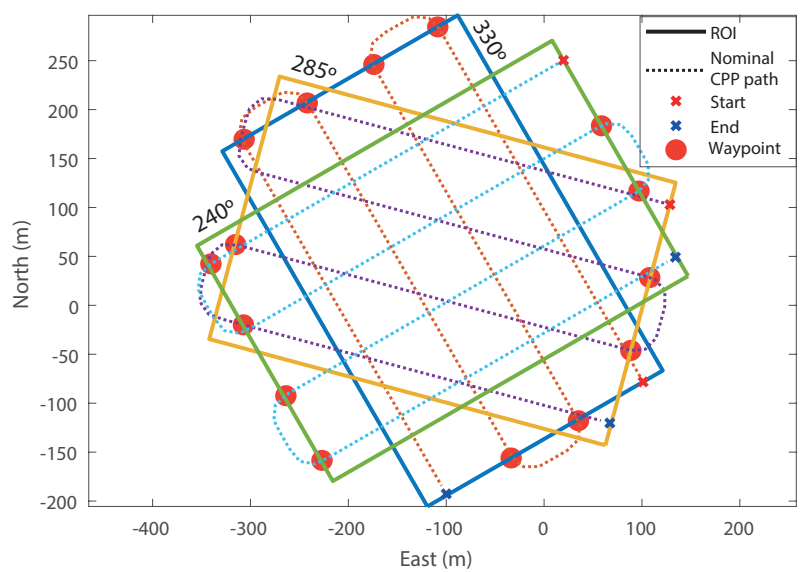

Fig. 3: Three ROI to be surveyed in flight testing with a nominal CPP for an example

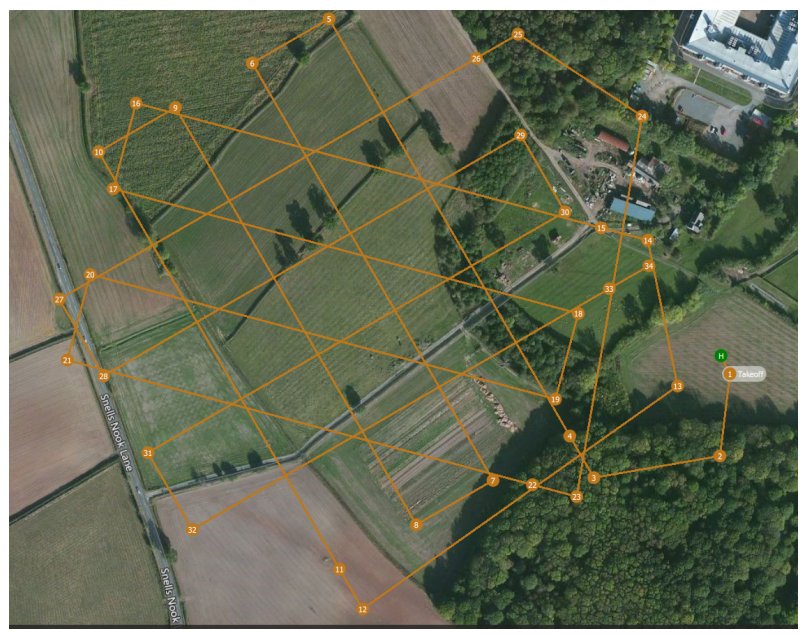

Fig. 4: Waypoints uploaded to X8 through QGroundControl

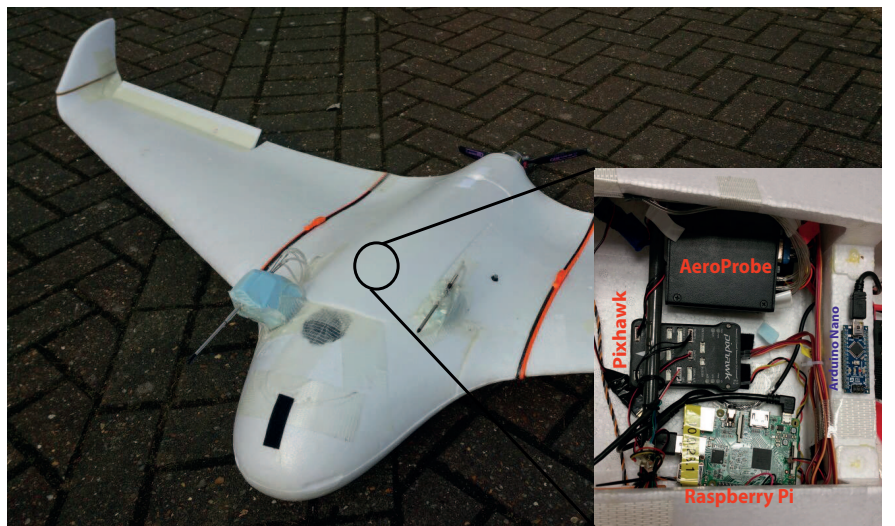

Fig. 5: The Skywalker X8 Flight test platform

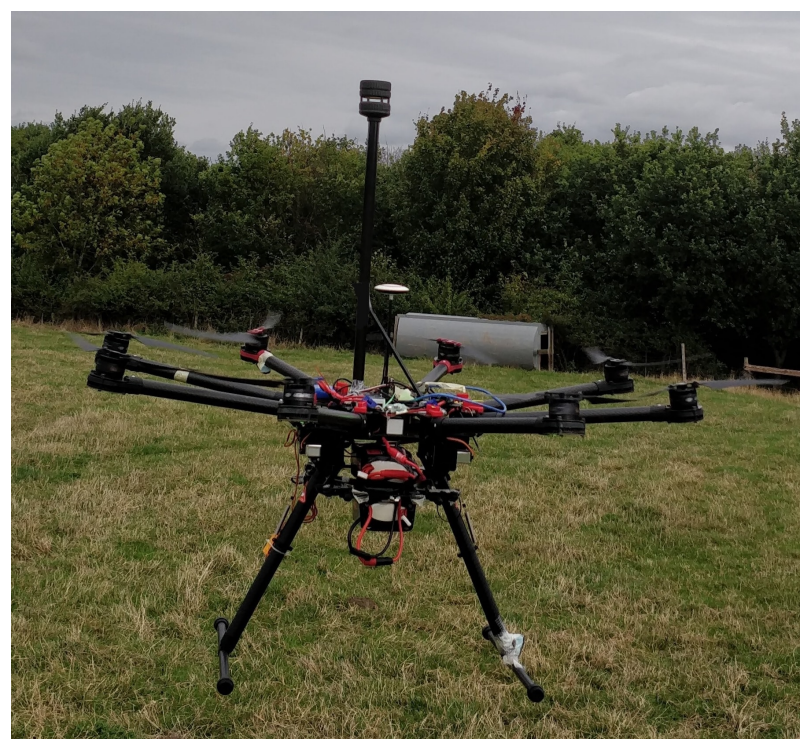

Fig. 6: S1000 with FT742 ultrasonic windspeed sensor mounted out of rotor wash

In the first mission the wind was $7 \mathrm{~m} / \mathrm{s}$ from $225^{\circ}$ with the reference airspeed of $16 \mathrm{~m} / \mathrm{s}$, this is an airspeed to wind ratio of $44 \%$. Taking the first survey (ROI polygon rotation angle $330^{\circ}$ ) from this mission as the example.

A comparison between flight paths, $V_{g}$, and distance travelled in the $330^{\circ}$ survey are shown in Fig. 7. The time taken in the actual flight was $145 \mathrm{~s}$, where the time predicted by the model is $147 \mathrm{~s}$, which is a very small $1.45 \%$ error. This accuracy is due the aircraft $V_{g}$ followed the prediction quite closely. Even in the transient turn phase, where the aircraft is still rolling to the required bank angle which is not modelled, it still follows closely. Which indicates that the instantaneous roll assumption is adequate for these small, low weight, low inertia, manoeuvrable aircraft.

The distance travelled by the aircraft in the survey was 
$2013 \mathrm{~m}$ compared to the predicted $1944 \mathrm{~m}$, this is a $4 \%$ distance error of $69 \mathrm{~m}$. This was caused by path overshoot due to non perfect path following.

It can be seen how significant wind can be on flight time. In the example survey the zero wind prediction is $123 \mathrm{~s}$, however the actual flight time is $147 \mathrm{~s}$ which is a significant difference of $16.4 \%$.

The remainder of the flight tests are presented in the following Table, which contains all the pertinent flight and model comparison data, for all three missions. This amounted to 13 surveys. The overall average time prediction accuracy is $2.84 \%$.

The final mission was performed in very extreme wind conditions for this size of aircraft, at a windspeed of $11 \mathrm{~m} / \mathrm{s}$ which is $69 \%$ of the it's airspeed. The overshoot error and thus the distance travelled error is greater, this led to slightly increased inaccuracy.

There was an anomalous result in the 5th survey of the 3rd mission where there was $5.52 \%$ error, where the median error for that mission is closer to $1.4 \%$. Seen in the Fig. 8 is the airspeed of the aircraft across this survey, and it can be seen that the airspeed tracking performance is poor in the turns. Meaning that the mean airspeed is higher than that of the 16 $\mathrm{m} / \mathrm{s}$ reference. By using $16 \mathrm{~m} / \mathrm{s}$ in the calculation the error can be brought down to $1.3 \%$. This shows that the prediction is dependant on accurate airspeed, and path tracking.

\section{A. Wind Field Measurements}

The wind field is measured for the 1st example mission, and then of three other ocastions with different wind conditions. The average measurements across the mission 1 wind survey were $7.3 \mathrm{~m} / \mathrm{s}$ and $230.2^{\circ}$. This closely matched the wind forecast information and was taken from a meteorological station 5 miles north of the test site which was $225^{\circ}$ at $7 \mathrm{~m} / \mathrm{s}$. The wind strength and direction remained quite consistent across the whole survey area and across the $14 \mathrm{~min}$ flight time, with standard deviations of $0.627 \mathrm{~m} / \mathrm{s}$ and $10.72^{\circ}$. The wind field with the S1000 flight path is shown on Fig. 9.

The other wind survey flights gave similarly consistent results, these can also be seen in Table 3. Weaker wind strength leads to slightly more variation in wind direction, however as the wind is weaker, this will have less effect on the aircraft.

The variations in the wind are small and thus will only have a small effect on the fight time prediction. To show this the mission 1 prediction is re-calculated with new wind, altered by the standard deviation of both wind direction and strength. By altering the wind direction by $\pm 10.72^{\circ}$ the predicted flight time varies from $145 \mathrm{~s}$ to $149.5 \mathrm{~s}$ which is a $3 \%$ variation. If the same is performed with a $\pm 0.67 \mathrm{~m} / \mathrm{s}$ wind speed the predicted time varies a similar small amount $144.5 \mathrm{~s}$ to $150 \mathrm{~s}$.

\section{CONCLUSION}

This author's previous work developed a model for calculating the flight time of a fixed wing aircraft during an
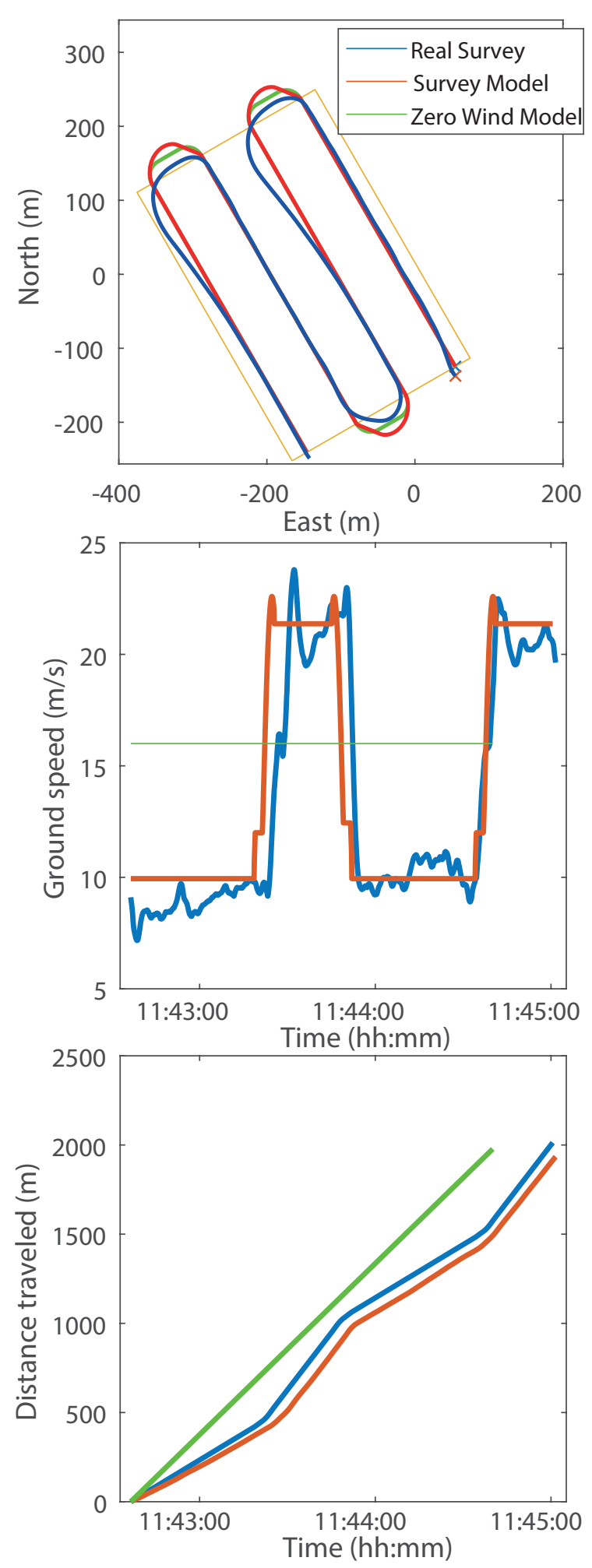

Fig. 7: Comparison between real survey flight with $\mathrm{X} 8$ and the survey model prediction at zero wind and the calculated wind that day for Mission 1 and a ROI rotation of $330^{\circ}$. Top: Show predicted versus actual aircraft paths, Middle: Ground speed, Bottom: distance travelled 
TABLE 2: Survey flight test compared to survey model results

\begin{tabular}{|c|c|c|c|c|c|c|}
\hline \# & Survey ROI Rotation & FTIW model time & Zero Wind Model Time & Flight Time & Error \% & Distance Error \\
\hline \multicolumn{2}{|r|}{ Mission 1} & \multicolumn{5}{|c|}{$V_{w}=7 \mathrm{~m} / \mathrm{s}, W_{d}=225^{\circ}, D_{x}=77.3 \mathrm{~m}$} \\
\hline 1 & Example $330^{\circ}$ & $147.3 \mathrm{~s}$ & 123.1 & $145.12 \mathrm{~s}$ & $1.48 \%$ & $69 \mathrm{~m}$ \\
\hline 2 & $285^{\circ}$ & $134.0 \mathrm{~s}$ & 123.1 & $134.5 \mathrm{~s}$ & $0.37 \%$ & $57.3 \mathrm{~m}$ \\
\hline 3 & $240^{\circ}$ & $128.6 \mathrm{~s}$ & $123.1 \mathrm{~s}$ & $128.6 \mathrm{~s}$ & $0.85 \%$ & $43.7 \mathrm{~m}$ \\
\hline \multicolumn{2}{|r|}{ Mission 2} & \multicolumn{5}{|c|}{$V_{w}=7.4 \mathrm{~m} / \mathrm{s}, W_{d}=335^{\circ}, D_{x}=77.3 \mathrm{~m}$} \\
\hline 1 & $330^{\circ}$ & $142.8 \mathrm{~s}$ & $123.1 \mathrm{~s}$ & $141.6 \mathrm{~s}$ & $0.84 \%$ & $103 \mathrm{~m}$ \\
\hline 2 & $285^{\circ}$ & $143.8 \mathrm{~s}$ & $123.1 \mathrm{~s}$ & $135.2 \mathrm{~s}$ & $6 \%$ & $99 \mathrm{~m}$ \\
\hline 3 & $240^{\circ}$ & $145.0 \mathrm{~s}$ & $123.1 \mathrm{~s}$ & $137.1 \mathrm{~s}$ & $5.4 \%$ & $87 \mathrm{~m}$ \\
\hline 4 & $240^{\circ}$ & $154.7 \mathrm{~s}$ & $123.1 \mathrm{~s}$ & $152.1 \mathrm{~s}$ & $1.68 \%$ & $89 \mathrm{~m}$ \\
\hline \multicolumn{2}{|r|}{ Mission 3} & \multicolumn{5}{|c|}{$V_{w}=11 \mathrm{~m} / \mathrm{s}, W_{d}=230^{\circ}, D_{x}=49.8 \mathrm{~m}$} \\
\hline 1 & $330^{\circ}$ & $201 \mathrm{~s}$ & $125 \mathrm{~s}$ & $195 \mathrm{~s}$ & $5.37 \%$ & $136.5 \mathrm{~m}$ \\
\hline 2 & $330^{\circ}$ & $201.6 \mathrm{~s}$ & $125 \mathrm{~s}$ & $192 \mathrm{~s}$ & $4.76 \%$ & $158.5 \mathrm{~m}$ \\
\hline 3 & $285^{\circ}$ & $200.1 \mathrm{~s}$ & $125 \mathrm{~s}$ & $197.3 \mathrm{~s}$ & $1.7 \%$ & $123.9 \mathrm{~m}$ \\
\hline 4 & $285^{\circ}$ & $200.1 \mathrm{~s}$ & $125 \mathrm{~s}$ & $197.7 \mathrm{~s}$ & $1.41 \%$ & $119 \mathrm{~m}$ \\
\hline 5 & $240^{\circ} @ \mathrm{~V}=16$ & $228.4 \mathrm{~s}$ & $125 \mathrm{~s}$ & $215.8 \mathrm{~s}$ & $5.52 \%$ & $95 \mathrm{~m}$ \\
\hline 5 & $240^{\circ} @ \mathrm{~V}=16.5$ & $213 \mathrm{~s}$ & $122.1 \mathrm{~s}$ & $215.8 \mathrm{~s}$ & $1.31 \%$ & $95 \mathrm{~m}$ \\
\hline 6 & $240^{\circ}$ & $213.3 \mathrm{~s}$ & $125 \mathrm{~s}$ & $210 \mathrm{~s}$ & $1.55 \%$ & $105 \mathrm{~m}$ \\
\hline
\end{tabular}

TABLE 3: Survey flight test compared to survey model results

\begin{tabular}{|c|c|c|c|c|c|c|}
\hline$\#$ & Wind Forecast & Average Wd & Average Vw & Std Wd & Std Vw & Height Flown \\
\hline 1 Example & $225^{\circ} / 7 \mathrm{~m} / \mathrm{s}$ & $230.2^{\circ}$ & $7.3 \mathrm{~m} / \mathrm{s}$ & $10.72^{\circ}$ & $0.627 \mathrm{~m} / \mathrm{s}$ & $100 \mathrm{~m}$ \\
2 & $240^{\circ} / 7.5 \mathrm{~m} / \mathrm{s}$ & $244.12^{\circ}$ & $8.3 \mathrm{~m} / \mathrm{s}$ & $9.62^{\circ}$ & $1.13 \mathrm{~m} / \mathrm{s}$ & $100 \mathrm{~m}$ \\
3 & $270^{\circ} / 3 \mathrm{~m} / \mathrm{s}$ & $289.3^{\circ}$ & $3.74 \mathrm{~m} / \mathrm{s}$ & $14.5^{\circ}$ & $0.518 \mathrm{~m} / \mathrm{s}$ & $100 \mathrm{~m}$ \\
4 & $270^{\circ} / 6.5 \mathrm{~m} / \mathrm{s}$ & $259.4^{\circ}$ & $7.08 \mathrm{~m} / \mathrm{s}$ & $10.1^{\circ}$ & $1.2 \mathrm{~m} / \mathrm{s}$ & $100 \mathrm{~m}$ \\
\hline
\end{tabular}

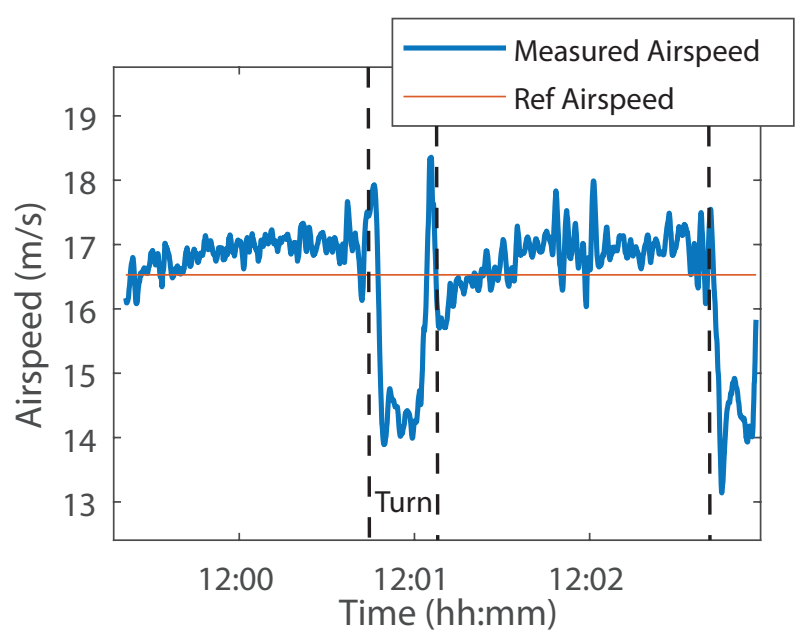

Fig. 8: Poor Airspeed tracking across survey, led to lower model accuracy

aerial survey. This is to be used as a critical tool to planning time optimal surveys in complex concave regions of interest. By performing a number of actual aerial surveys with a representative aircraft in different wind conditions, the model accuracy is further tested and validated.

The model had a low overall time prediction inaccuracy of $2.84 \%$. It has also been demonstrated that to achieve these levels of accuracy, both the airspeed and path following tracking performance must be reasonable, but shown to be achievable with a low cost COTS system.

It was also found that the steady uniform wind assumption holds over short missions. While there was some variation in wind over the flight, it was shown to be small and also demonstrates that these variations would only have minimal

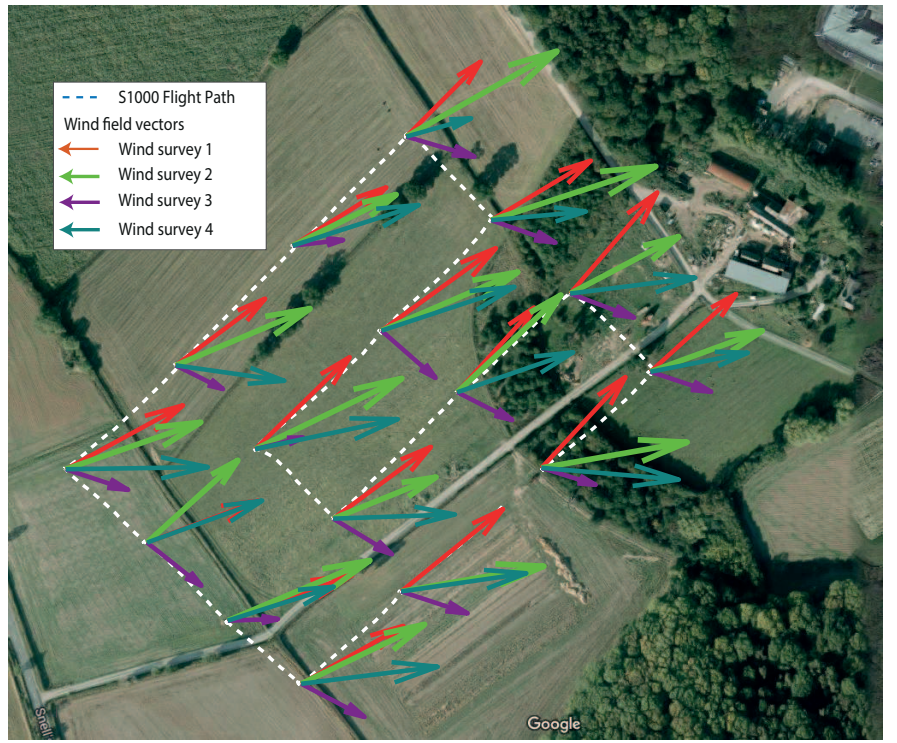

Fig. 9: Windspeed and direction wind field at wind sample locations for 4 different wind measurement flights

impact on the survey flight time.

As this tests are on a small scale and on simple polygons, in the future these test will be scaled up to a full survey on a more complex concave ROI.

\section{ACKNOWLEDGEMENT}

This work was supported by Science and Technology Facilities Council (STFC) under Newton Fund with grant number ST/N006852/1, and by the EPSRC with grant number EP/P012868/1. 


\section{REFERENCES}

[1] World Bank. About the agriculture global practice. Technical report, World Bank Group, 2014.

[2] David J. Mulla. Twenty five years of remote sensing in precision agriculture: Key advances and remaining knowledge gaps. Biosystems Engineering, 114(4):358 - 371, 2013. Special Issue: Sensing Technologies for Sustainable Agriculture.

[3] R Gini, D Pagliari, D Passoni, L Pinto, G Sona, and P Dosso. Uav photogrammetry: Block triangulation comparisons. Int. Arch. Photogram. Remote Sens. Spat. Inf. Sci, XL-1/W2:pp.157-162, 2013.

[4] Kimberly M Fornace, Chris J Drakeley, Timothy William, Fe Espino, and Jonathan Cox. Mapping infectious disease landscapes: unmanned aerial vehicles and epidemiology. Trends in parasitology, 30(11):514 519, 2014.

[5] Juliane Bendig, Andreas Bolten, Simon Bennertz, Janis Broscheit, Silas Eichfuss, and Georg Bareth. Estimating biomass of barley using crop surface models (csms) derived from uav-based rgb imaging. Remote Sensing, 6(11):10395-10412, 2014.

[6] Howie Choset. Coverage of known spaces: The boustrophedon cellular decomposition. Autonomous Robots, 9(3):247-253, 2000.

[7] A. Xu, C. Viriyasuthee, and I. Rekleitis. Optimal complete terrain coverage using an unmanned aerial vehicle. In 2011 IEEE International Conference on Robotics and Automation, pages 2513-2519, May 2011.

[8] Timo Oksanen and Arto Visala. Coverage path planning algorithms for agricultural field machines. Journal of Field Robotics, 26(8):651-668, 2009.

[9] Ardupilots webpage. http://ardupilot.org/planner/. Accessed on Sept 2018.

[10] Qgroundcontrol's website. http://qgroundcontrol.com/. Accessed on Sept 2018.

[11] Dji planner's website. http://www.djiflightplanner.com/. Accessed on Sept 2018.

[12] Cunjia Liu Matthew Coombes, Wen-Hua Chen. Fixed wing uav survey coverage path planning in wind for improving existing ground control station software. In 37th Chinese Control Conference, 2018.

[13] R. N. De Carvalho, H. A. Vidal, P. Vieira, and M. I. Ribeiro. Complete coverage path planning and guidance for cleaning robots. In Industrial Electronics, 1997. ISIE '97., Proceedings of the IEEE International Symposium on, volume 2, pages 677-682 vol.2, Jul 1997.

[14] Yan Li, Hai Chen, Meng Joo Er, and Xinmin Wang. Coverage path planning for $\{\mathrm{UAV}\}$ based on enhanced exact cellular decomposition method. Mechatronics, 21(5):876 - 885, 2011. Special Issue on Development of Autonomous Unmanned Aerial Vehicles.

[15] W. H. Huang. Optimal line-sweep-based decompositions for coverage algorithms. In Proceedings 2001 ICRA. IEEE International Conference on Robotics and Automation (Cat. No.01CH37164), volume 1, pages 27-32 vol.1, 2001.

[16] Matthew Coombes, Tom Fletcher, Wen-Hua Chen, and Cunjia Liu. Optimal polygon decomposition for uav survey coverage path planning in wind. Sensors, 18(7), 2018.

[17] Arthur Richards. Flight optimization for an agricultural unmanned air vehicle. In European Control Conference (ECC), 2018.

[18] M. Coombes, W. H. Chen, and C. Liu. Boustrophedon coverage path planning for uav aerial surveys in wind. In 2017 International Conference on Unmanned Aircraft Systems (ICUAS), pages 15631571, June 2017.

[19] Laszlo Techy and Craig A Woolsey. Minimum-time path planning for unmanned aerial vehicles in steady uniform winds. Journal of guidance, control, and dynamics, 32(6):1736-1746, 2009.

[20] Matthew Coombes, Wen-Hua Chen, and Peter Render. Landing site reachability in a forced landing of unmanned aircraft in wind. Journal of Aircraft, pages 1-13, February 2017.

[21] S. Schopferer and T. Pfeifer. Performance-aware flight path planning for unmanned aircraft in uniform wind fields. In 2015 International Conference on Unmanned Aircraft Systems (ICUAS), pages 11381147, June 2015.

[22] Nicola Ceccarelli, John J Enright, Emilio Frazzoli, Steven J Rasmussen, and Corey $\mathrm{J}$ Schumacher. Micro uav path planning for reconnaissance in wind. In American Control Conference, 2007. ACC'07, pages 5310-5315. IEEE, 2007.

[23] A. L. Jennings, R. Ordonez, and N. Ceccarelli. Dynamic programming applied to uav way point path planning in wind. In 2008 IEEE International Conference on Computer-Aided Control Systems, pages 215-220, Sept 2008.
[24] Sanghyuk Park, John Deyst, and Jonathan P How. A new nonlinear guidance logic for trajectory tracking. In AIAA guidance, navigation, and control conference and exhibit, pages 16-19, 2004.

[25] Jean Smith Mike Hutchison Pawel Ladoz, Matthew Coombes. Robot Operating System (ROS), volume 778. Springer, 2018. 